\title{
Design of novel screening environments for Mild Cognitive Impairment: giving priority to elicited speech and language abilities
}

\author{
Sofia Segkouli ${ }^{1,2,}$ Ioannis Paliokas ${ }^{1}$, Dimitrios \\ Tzovaras ${ }^{1}$, Dimitrios Giakoumis ${ }^{1}$ \\ ${ }^{1}$ Information Technologies Institute-ITI \\ Centre for Research and Technology Hellas- \\ CERTH, Thessaloniki, Greece \\ sofia.segouli@gmail.com, ipaliokas@iti.gr, \\ dimitrios.tzovaras@iti.gr, dgiakoum@iti.gr
}

\author{
Charalampos Karagiannidis ${ }^{2}$ \\ ${ }^{2}$ Department of Special \\ Education \\ University of Thessaly, \\ Volos, Greece \\ karagian.uth@gmail.com
}

\begin{abstract}
Recent cognitive decline screening batteries have highlighted the importance of language deficits related to semantic knowledge breakdown to reveal the incipient dementia. This paper proposes the introduction of novel enriched linguistic tests and examines the hypothesis that language can be a sensitive cognitive measure for Mild Cognitive Impairment (MCI). A group of MCI and healthy elderly were administered a set of proposed linguistic tests. Performance measures were made on both groups to indicate that concrete verbal production deficits such as impaired verb fluency can distinguish the MCI from normal aging. In addition, it was found that even in cases where the MCI subjects preserved scores, language tests took significantly more time compared to healthy controls. These findings indicate that language could be a sensitive cognitive marker in preclinical stages of MCI.
\end{abstract}

Keywords-component; Mild Cognitive Impairment, Screening batteries, Linguistic Test, Verbal fluency

\section{INTRODUCTION}

Language deficits are an important diagnostic criterion of early cognitive changes that occur both in Alzheimer Disease (AD) and Mild Cognitive Impairment (MCI). Understanding language performance in MCI facilitates the process of selecting tasks in screening tests with diagnostic and prognostic value [1]. Also, subtle semantic memory impairments could be considered as early markers of MCI to AD progression [2].

Related research efforts have so far focused on language tests for the initial stages of $\mathrm{AD}$ with respect to the single word lexical retrieval and the assessment of textual reading and lexical production paradigms [3], [4]. Most of them overemphasize memory dysfunction [5] to the neglect of problems arising exclusively from language [6]. Screening batteries are limited in the utilization of confrontation naming tasks and word generation tasks [7]. The Boston Naming Test (BNT) [8] assesses single word confrontation naming, while in the Montreal Cognitive Assessment (MoCa) test [9] language is assessed through a three-item confrontation naming task with low-familiarity animals, repetition of two syntactically complex sentences and the fluency task. Moreover, free storytelling or retelling of stories such as the Cookie Theft Stimuli (CTS) [10] targets to record the spontaneous speech of patients with MCI and Alzheimer through pictures stimuli.
Linguistic and cultural factors are liable for a smaller portion of language subtests in computerized screening batteries [11]. However, a deeper analysis of language deficits in screening batteries is missing. Thus the present study aims to explore the effect of language and identify new more complex linguistic alternatives for detecting deficits in early dementia.

\section{SEMANTIC AND LEXICAL RETRIEVAL TESTS FOR MCI ASSESSMENT}

\section{A. MCI Screening Tests and Computerized Batteries}

Various computerized MCI screening tests exist as adaptations or combinations of the standardized neuropsychological tests [12]. Verbal fluency tasks are included in neuro-psychological measures of older adults but the linguistic part is partly examined or completely absent [13]. New computerized tests [14], [15] as the CANS-MCI (The Computer-Administered Neuropsychological Screen for MCI) focus on the fluency factor (words' recall and sentence repetition) [16]. Words' production is related to letter and category fluency, while grammatical and syntactic processing along with semantic breakdown is not yet examined systematically in screening batteries.

Moreover, deficits in verb and action processing have been examined [17], but not fully exploited in screening batteries. Narrative recall has been proved more effective than the Mini Mental State Examination Test (MMSE) [18] in recognizing MCI clinical syndrome, but still considered insufficient for adequate discrimination between healthy and impaired subjects.

The main aim of the present paper is to introduce a new set of exercises that will leverage the importance of lexical production by elderly subjects in a more realistic context. The following sections present indicatively some examples of the language test bank that we have developed.

\section{B. Enriching the Linguistic part of Screening Tests}

A novel computerized environment enhanced with linguistic characteristics which implicate semantic memory has been constructed. The validity of the proposed novel tests was compared with that of traditional neuropsychological tests. In 
this scope, well-established tests as the MoCa and BNT tests have also been transferred in our computerized environment for automation and individual's progress monitoring.

The current designs of the proposed computerized screening batteries encompass language skills such as naming of pictures and objects in isolation from everyday discourse [17] [18]. The common task of picture naming is dependent on single word recognition its description and more complex narrative or spontaneous discourse. The picture description task can sensitively detect complex communication abilities [19]. A picture sequence encourages narration through temporal and logical sequence of events while a single picture prompts only a single description. However, narration could be time consuming while single picture confrontation naming could limit the information about semantic deficits of MCI.

The proposed lexico-semantic subtests intend to assess language in a different notion, oriented by combined cognitive functions, such as semantic ability, lexical production, executive function, mental control and spatial ability. For this purpose, the confrontation naming performance measured by BNT was compared to free naming in an imaging environment with conceptual consequence in order to define the difficulty in interaction between the semantic and lexical production. Multiple and equivalent formats have been used to result in a reduction in practice effects.

\section{ACtion NAMING AND SEMANTIC PROCESSING}

It is assumed that people having MCI face difficulties in naming, visual perception and the ability to understand pictorial information. The picture description task is commonly used in patients with early aMCI and AD to detect subtle language deficits and conceptualize how memory changes [20] [21], [22]. Still there is need for language assessment that reflects real-world and everyday life situations. The following tests are proposed in the present paper: i) Naming Actions ii) Pictures to Sentences iii) Matching Categories.

\section{A. Research Hypothesis in Naming ability}

To test our hypotheses, the first test of a defined language bank, called 'Naming Actions' uses images as 'vehicles', to depict people performing everyday actions. Participants have to name these tasks, without time limits or help provided during the test. One single example was given prior the subtest to familiarize participants with task demands.

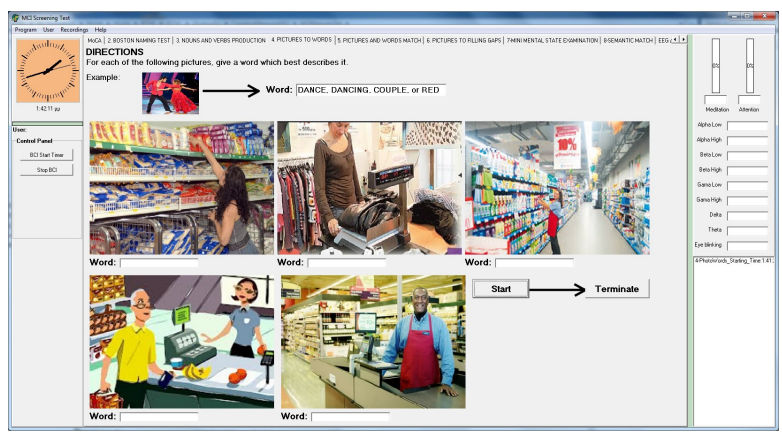

Fig. 1. Screenshoot of the 'Naming Actions' test
This test of our screening battery examines the naming ability (Fig 1). The aim was to construct a picture -supported task with complexity in its performance. Four conceptually related photos and one irrelevant were given for action naming -instead of abstract objects or sketches- so as to involve multiple cognitive function domains in the assessment. Participants have to find the temporal sequence and afterwards retrieve the concrete verb in order to describe the action. The photos are presented in a competitive manner to increase difficulty on decision making. The tested hypotheses are:

- HAl: MCI patients need more time to complete the naming tasks than healthy elderly.

- HA2: MCI patients use fewer verbs than the healthy elderly when naming actions.

\section{B. Research Hypothesis in Sentences and Semantically Related Pictures}

The aim of this exercise is to enrich the naming task with linguistic implications (Fig 2). The perceived difficulty lies in the need to combine cognitive and linguistic abilities for performing successfully the tests. Test givers have to match the meaning of pictures with equal number of filling gaps in sentences, so that the sentences preserve a meaning related to one selected picture, be grammatically correct and keep their internal coherence. The sentences are semantically independent from each other. This test was designed to examine the following hypotheses:

- HB1: MCI patients need more time than healthy elderly to complete the sentences.

- HB2: MCI patients score lower than the healthy elderly when filling in the sentences with picture stimuli.

\section{Research Hypothesis in Matching Categories}

This novel subtest, which involves matching words with the proper semantically related category, examines the difficulty to recognize the semantic distance between groups of words to create a free number of categories by them. Participants in this test try to match similar words and create groups (Fig 3). The number of word-groups is not predefined and can change during the test. Reaction time was considered as a potentially critical parameter correlating with semantic deficits appearance.

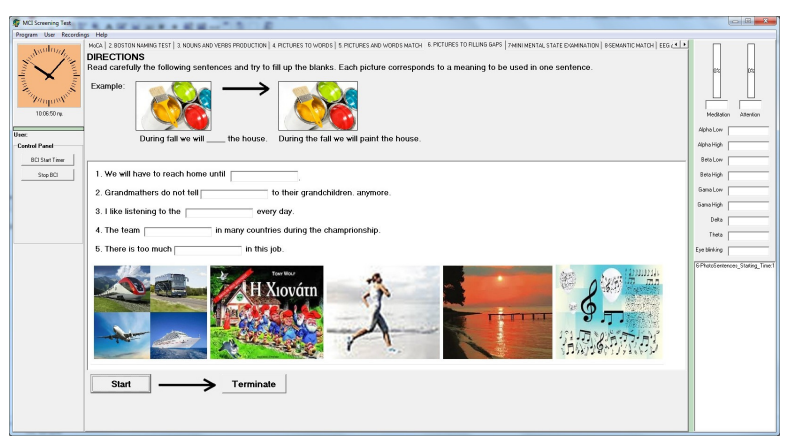

Fig. 2. Screenshoot of the 'Pictures to Sentences' test 


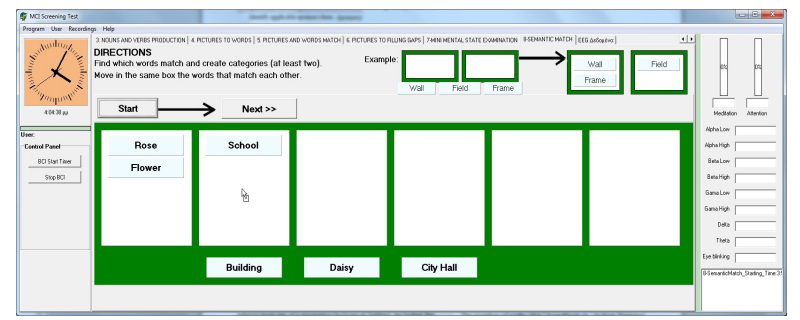

Fig. 3. Screenshot of the 'Matching Categories' test

MCI patients were expected to face difficulties in matching words which are semantic relatives. The hypotheses for this task are as follows:

- HC1: MCI patients need more time to match words (categorize) than the healthy elderly.

- HC2: MCI patients score lower in the word matching test by creating less correct matches.

\section{Experimental Evaluation and Methodology}

A pilot study was performed to test the above hypotheses toward retaining the proposed tests as a part of the overall MCI screening test development. The participants underwent multiple administrations of a neuropsychological battery that included the BNT, the Moca fluency task and afterwards the above described novel semantic, lexical retrieval tests. Subjects were tested a few times spaced over a fixed time span. All procedures were performed in accordance with ethical protocols approved by the hosted institute.

Two groups were enrolled in this study: a. healthy elderly and b. MCI users. Recruitment criteria included at least 55 years of age, being self-handlers and having basic computer skills. Twenty five $(\mathrm{N}=25)$ elderly persons aged from 55 to 78 years old $(\mathrm{M}=65.57, \mathrm{SD}=5.89)$ composed the testing group. Approximately $40 \%(\mathrm{~N}=10)$ of the elderly were MCI positives, non-demented. The MCI group was closely matched with healthy elderly in terms of age, education level, gender, selfhandling abilities and computer skills. All tests were performed in Greek language. Time performance was automatically calculated by time-stamping the user's responses in log files.

\section{The Linguistic Test Assessment Results}

First of all, according to Pearson's method, a significant correlation between the computerized and the 'paper and pencil' BNT test results $(\mathrm{r}=0.95, \mathrm{p}<0.001)$ was found. In turn, these results impact on the computer-based test validity of using its scores as cognitive decline reference for comparisons with the novel tests.

Linguistic MoCa results for healthy elderly $(\mathrm{M}=13.80$, $\mathrm{SD}=4.94)$ were found significantly different $(\mathrm{t}=-2.29, \mathrm{p}<0.05)$ than for those having MCI $(\mathrm{M}=9.10, \mathrm{SD}=4.84)$. The ShapiroWilk test showed significant deviance from normality for BNT results, so non-parametric tests were used. Among elderly users, those having MCI $(\mathrm{M}=21.78, \mathrm{SD}=5.40)$ had significantly different BNT results $(\mathrm{p}<0.05)$ than the healthy ones $(\mathrm{M}=25.90$, $\mathrm{SD}=1.97)$. Concerning the BNT scores in relation to the age of participants, the Kruskal-Wallis test indicated that normal aging does not affect the results $(\mathrm{p}>0.05)$. Thus, a valid comparison among the paper-based tests' results and the proposed linguistic subtests was made. Ideally, linguistic test results were compared to those extracted from the BNT and $\mathrm{MoCa}$ and minimum differences were found in their ability to distinguish the MCI from normal elderly, as explained in the following.

The results of the first test ('Naming Actions') indicated that the MCI group needed considerably more time (HA1 Hypothesis) to name the performed actions in pictures $(\mathrm{M}=44.39 \mathrm{sec}, \mathrm{SD}=12.39)$ than the control group $(\mathrm{M}=32.56$, $\mathrm{SD}=10.23)$ and this difference was found significant $(\mathrm{t}=-3.7$, $\mathrm{p}<0.001)$. The 'Pictures to Sentences' test gave strong differences in task durations (Mann-Whitney $\mathrm{U}=50.00$, $\mathrm{p}=$ 0.011 ), with $72.88 \mathrm{sec}(\mathrm{SD}=47.16)$ for the healthy elderly and $148.77 \mathrm{sec}(\mathrm{SD}=116.91)$ for the MCI (HB1 Hypothesis). Similarly, significant difference $(\mathrm{t}=-2.9, \mathrm{p}<0.05)$ was found regarding the duration of the 'Matching categories' test, where normal aged persons needed $29.26 \mathrm{sec}(\mathrm{SD}=14.99)$ to complete the test, in contrast with people having MCI who needed 38.38 $\sec (\mathrm{SD}=16.26)$ in average respectively (HB1 Hypothesis).

All duration variables of the three tests gave a promising silhouette measure of cohesion and separation $(\sim 0.75)$ and this indicates that it is possible that the proposed linguistic test assessments can be included in a future MCI screening test as they contribute to the significance of the clustering model.

The second part of the analysis is targeted on the number of correct responses, so as to complete the use case analysis of the proposed linguistic tests. Regarding the MCI group, the number of verbs (HA2 hypothesis in 'Naming Actions' test), the number of properly filled sentences (HB2 hypothesis in 'Pictures to Sentences') and the number of correct matches (HC2 hypothesis in 'Matching Categories' test) were not found significantly different than those of the control group.

Results so far support the hypothesis that older people preserve performance while being relatively slower than young adults [23] [24] [25]. However, a possible extension of these findings to include MCI could possibly be cross-checked in the future by using $\alpha$ larger population. Currently, the evidence upgrades the role of time [26] as a promising factor for evaluating the linguistic tests, whereas subtests' duration in our proposed tests can been seen as a valuable indication to assess MCI. Table 1 provides an overview of the main findings of the above analyses.

It is worth mentioning that the BNT results were found linearly related to the linguistic task durations and this could be proved valuable for the MCI patient's monitoring. A medium strength of association between the computerized BNT scores and the durations of the 'Naming Actions' test was found $(r(23)=-0.46, p<0.013)$. A larger strength was found for the 'Matching Categories' test with $\mathrm{r}(23)=-0.52, \mathrm{p}=0.005$, while even better results came from the 'Pictures to Sentences' test with $\mathrm{r}(23)=-0.62, \mathrm{p}<0.001$. The correlation of the three tests with the linguistic MoCa scores were found moderate $\mathrm{r}=[-0.35$, -0.42] with $\mathrm{p}<0.05$. Conclusively the decline in linguistic abilities seems to be stepped enough and linearly related to the memory retrieval, which is tested by the traditional screening tests. Thus, linguistic tests can replicate memory tests when needed in MCI screening administration. 


\section{TABLE 1. MCI SCREENING TEST RESULTS}

\section{Parameters}

\begin{tabular}{lll}
\multicolumn{1}{c}{ Parameters } & \multicolumn{2}{c}{ Groups of participants } \\
& $\begin{array}{r}\text { Healthy Elderly } \\
\text { Sex M/F (in \%) }\end{array}$ & \multicolumn{1}{c}{ MCI } \\
Age (in years) & $63 / 33$ & $60 / 40$ \\
Education (in years) & $13.46(\mathrm{SD}=3.33)$ & $12.9(\mathrm{SD}=3.38)$ \\
Linguistic MoCa & $13.80(\mathrm{SD}=4.94)$ & $9.1(\mathrm{SD}=4.84)$ \\
$\begin{array}{l}\text { Computerized BNT results } \\
\text { (without help) }\end{array}$ & $26.00(\mathrm{SD}=1.18)$ & $21.13(\mathrm{SD}=5.38)$ \\
$\begin{array}{l}\text { Computerized BNT results } \\
\text { (with semantic help) }\end{array}$ & $26.27(\mathrm{SD}=1.38)$ & $21.63(\mathrm{SD}=5.12)$ \\
$\begin{array}{l}\text { Computerized BNT results } \\
\text { (with phonemic help) }\end{array}$ & $26.27(\mathrm{SD}=1.38)$ & $22.00(\mathrm{SD}=5.04)$ \\
Computerized BNT duration $(\mathrm{sec})$ & $116.67(\mathrm{SD}=21.62)$ & $176.73(\mathrm{SD}=70.43)$ \\
Naming Actions duration $(\mathrm{sec})$ & $32.56(\mathrm{SD}=10.23)$ & $44.39(\mathrm{SD}=12.39)$ \\
Pictures to Sentences duration $(\mathrm{sec})$ & $72.88(\mathrm{SD}=47.16)$ & $148.77(\mathrm{SD}=116.91)$ \\
Matching Categories duration $(\mathrm{sec})$ & $29.26(\mathrm{SD}=14.99)$ & $38.38(\mathrm{SD}=16.26)$
\end{tabular}

\section{CONCLUSION}

The main conclusion drawn from the present study is that novel interactive linguistic components, namely proper words' production and semantic ability associated with executive function and processing speed can be used as an instrument for early detection of MCI. Future studies should focus on the implementation of tests assessing the language ability at different levels of screening: word level, sentence level, discourse level within tasks' performance from real life conditions. Moreover, complex language deficits such as constructing a sentence in various syntactic forms, producing and matching successfully words with their semantic meaning as well as examining the individual prosodic cues, mood (particularly symptoms of depression), as well as emotional features may provide powerful information with diagnostic and therapeutic utility for dementia as another useful indicative marker for early intervention. These innovative linguistic tests may be proved a valuable tool in health care administration, for future MCI assessment to support screening procedures.

\section{ACKNOWLEDGEMENT}

This work has received funding from the European Union's 7 F.P.-Collaborative Project Prosperity4All (CP) under grant agreement No 610510.

\section{REFERENCES}

[1] V. Taler \& N.A. Phillips, Language performance in Alzheimer's disease and mild cognitive impairment: a comparative review. J. of Clinical \& Experimental Neuropsychology, 30(5), pp. 501-556, 2008.

[2] C. Borg, et al. "Semantic knowledge of newly coined words in mild cognitive impairment and Alzheimer's disease." Journal of Neurolinguistics v. 25(4), pp. 263-275, 2012.

[3] J. Rinaldi, G. Sbardelloto, C.H. Kristensen \& M.A.D.M.P. Parente, Textual reading comprehension and naming in Alzheimer's disease patients. Dementia \& Neuropsychologia, v. 2(2), 131-138, 2008.

[4] LL. Mansur, "Language Alterations in Alzheimer's Disease and other Dementias." Language Disturbances in Adulthood: New Advances from the Neurolinguistics Perspective. Sharjah, United Arab Emirates (UAE): Bentham Science Publishers, pp. 59-70, 2011.
[5] R. Thornton \& L.L. Light, Language comprehension and production in normal aging. Handbook of the psychology of aging, v. 6, pp. 261287, 2006.

[6] B. Cullen, B. O'Neill, J.J., Evans, R.F.Coen \& B.A. Lawlor. A review of screening tests for cognitive impairment. J. Neurol, Neurosurg Psychiatry, v. 78(8), pp. 790-799, 2007.

[7] L.K. Obler \& S. Pekkala, Language and communication in aging. Handbook of neurolinguistics, pp. 351-358, 2008.

[8] F.R. Ferraro \& K. Lowell, Boston Naming Test. Corsini Encyclopedia of Psychology, 2010.

[9] Z.S. Nasreddine et al. "The Montreal Cognitive Assessment, MoCA: a brief screening tool for mild cognitive impairment." Journal of the American Geriatrics Society, v. 53(4), pp. 695-699, 2005.

[10] R.H. Hamilton, et al. "Stimulating conversation: enhancement of elicited propositional speech in a patient with chronic non-fluent aphasia following transcranial magnetic stimulation", Brain and language, v. 113(1), pp. 45-50, 2010.

[11] C. Parker \& I.Philp, "Screening for cognitive impairment among older people in black and minority ethnic groups", Age and ageing, 2004.

[12] S. Zygouris \& M. Tsolaki "Computerized Cognitive Testing for Older Adults A review", American journal of Alzheimer's disease and other dementias, 2014, poi:1533317514522852.

[13] Z. Shao, E. Janse, K. Visser \& A.S. Meyer, What do verbal fluency tasks measure? Predictors of verbal fluency performance in older adults, Frontiers in psychology, v. 5, 2014.

[14] K. Wild, D. Howieson, F. Webbe, A. Seelye, J. Kaye, The Status of Computerized Cognitive Testing in Aging: A Systematic Review, Alzheimers Dement, v. 4(6), pp. 428-437, 2008.

[15] T. Dwolatzky, et al. "Validity of a novel computerized cognitive battery for mild cognitive impairment", BMC geriatrics, v. 3(4), 2003.

[16] J.B. Tornatore, E. Hill, J.A. Laboff \& M.E. McGann, Selfadministered screening for mild cognitive impairment: initial validation of a computerized test battery, J Neuropsychiatry Clin Neurosci, v. 17(1), pp. 98-105, 2005.

[17] T.H. Bak, et al. "Clinical, imaging and pathological correlates of a hereditary deficit in verb and action processing", Brain, v. 129(2), pp. 321-332, 2006.

[18] B. Roark, M. Mitchell, J.P. Hosom, K. Hollingshead \& J. Kaye,. Spoken language derived measures for detecting mild cognitive impairment.Audio, Speech, and Language Processing, IEEE Transactions on, v. 19(7), pp. 2081-2090, 2011.

[19] Y. Faroqi-Shah \& C.K. Thompson, Effect of lexical cues on the production of active and passive sentences in Broca's and Wernicke's aphasia, Brain and Language, v. 85(3), pp. 409-426, 2003.

[20] H. Choi, Performances in a picture description task in Japanese patients with Alzheimer's disease and with Mild Cognitive Impairment, Korean J Commun Disord, v. 14, pp. 326-337, 2009.

[21] K.E. Nutter-Upham et al. "Verbal fluency performance in amnestic MCI and older adults with cognitive complaints." Archives of Clinical Neuropsychology, v. 23(3), pp. 229-241, 2008.

[22] B.A. Ally, Using pictures and words to understand recognition memory deterioration in amnestic Mild Cognitive Impairment and Alzheimer's Disease: a review. Current neurology and neuroscience reports, v. 12(6), pp. 687-694, 2012.

[23] E. Tsantali, D.Economidis \& M. Tsolaki, Could language deficits really differentiate Mild Cognitive Impairment (MCI) from mild Alzheimer's disease?, Archives of gerontology and geriatrics, v. 57(3), pp. 263-270, 2013.

[24] J.D. Rohrer, W.D. Knight, J.E. Warren, N.C. Fox, M.N. Rossor \& J.D. Warren, Word-finding difficulty: a clinical analysis of the progressive aphasias. Brain, v. 131(1), pp. 8-38, 2008.

[25] E.L. Glisky, Changes in cognitive function in human aging. Brain aging: models, methods, and mechanisms, pp. 3-20, 2007.

[26] I. Tarnanas, M. Tsolaki, T. Nef, M Müri, R. \& U.P. Mosimann, Can a novel computerized cognitive screening test provide additional information for early detection of Alzheimer's disease?, Alzheimer's \& Dementia, v. 10(6), pp. 790-798, 2014. 